\title{
Posterior Polar Cataract and Its Management
}

\author{
Smita Anand ${ }^{1,2}$, Nimmi Rani1,2 \\ ${ }^{1}$ Aravind Eye Hospital, Madurai, India \\ ${ }^{2} \mathrm{PG}$ Institute of Ophthalmology, Madurai, India \\ Email: "smita.anand2001@gmail.com
}

Received 28 February 2015; accepted 13 July 2015; published 16 July 2015

Copyright (C) 2015 by authors and Scientific Research Publishing Inc.

This work is licensed under the Creative Commons Attribution International License (CC BY).

http://creativecommons.org/licenses/by/4.0/

C) (i) Open Access

\begin{abstract}
Although cataract surgery in posterior polar cataract is associated with posterior capsular rent and nucleus drop, by taking precautions the rate of complication can be decreased. The aim of this article is to review the etiology, pathogenesis, clinical features, and management of posterior polar cataract.
\end{abstract}

Keywords

Posterior Polar Cataract, Posterior Capsular Rent, Nucleus Drop

\section{Introduction}

Posterior polar cataract (PPC) is a relatively uncommon form of congenital cataract accounting for around $0.5 \%$ to $2 \%$ of the total cataract. It presents a special challenge to cataract surgeon because of high likelihood of posterior capsular rent (PCR) and nucleus drop during surgery. Although incidence of posterior capsular dehiscence has been reported to be $26 \%-36 \%$ [1] [2] in older studies, its incidence has been reduced to $7.1 \%-16.4 \%$ [3] according to recent study.

Incidence-Incidence of PPC ranges from 3 to 5 in 1000 [4]. It is found to be bilateral in $65 \%-80 \%$ of the cases [2] [5]. There is no sex predilection in general.

Morphology - Posterior polar cataract is a variant of developmental cataract. The lens may have a small opacity at birth. However, the cataractous changes usually occur later in life. It appears as a white dense opacity located in the posterior cortex, posterior capsule and the posterior subcapsular region. These cataracts have a different appearance compared with the routine posterior subcapsular axial opacity that occurs in young adults. There are onion-like concentric rings around the central opacity (bull's eye).

"Corresponding author.

How to cite this paper: Anand, S. and Rani, N. (2015) Posterior Polar Cataract and Its Management. Surgical Science, 6, 304-310. http://dx.doi.org/10.4236/ss.2015.67046 
Inheritance and genetics - It has been recognized that ppc seems to follow an autosomal dominant inheritance pattern [6], although it is occasionally sporadic. Positive family history was found in $40 \%-55 \%$ of the patients [1] [2]. Molecular genetic analyses have demonstrated that an autosomal-dominant posterior polar cataract is a genetically heterogenous disease [6]. The direct cause of the lenticular fiber malformation during lens development has not been well understood. There are five genes attributed to posterior polar cataract (CTTP) that have been identified. CTPP1 (OMIM 116600) has been mapped to 1p36 [6]. CTPP2 has been associated with CRYAB on 11q22-q22.3, and a Pro20Ser mutation and a deletion mutation (450delA) have also been highlighted [7]. The CHMP4B gene on chromosome 20p12-q12 is responsible for CTPP3 (OMIM 605387). Three mutations of PITX3 gene on chromosome 10q25, 38G > A mutation, 17-bp insertion, and 650delG have been reported to cause CTPP4 (OMIM 610623) [6].

\section{Pathogenesis}

The position of lens opacity is largely determined by the anatomy of the lens and the timing and nature of the insult that caused the abnormality by altering the embryogenesis. It has been suggested that posterior polar cataracts are caused by persistence of the hyaloid artery [5] or invasion of the lens by mesoblastic tissue. It appears that posterior polar cataract forms during embryonic life or early in infancy and usually becomes symptomatic 30 - 50 years later. The exact pathogenesis of posterior polar cataract is still unknown. However, it has been noted to occur as a result of gene mutation (6). A posterior polar cataract consists of dysplastic lens fibers, which, in their migration posteriorly from the lens equator, exhibit progressive lens opacity, increased degenerative changes, with the formation of a characteristic discoid posterior polar plaque-like cataract and the accumulation of extracellular material [8]. It seems that the high incidence of posterior capsule rupture during surgery for those patients might be because of two reasons. First, there might be tight adherence of the plaque to an otherwise normal capsule. Second, the posterior capsule itself underlying the plaque is exceptionally thin that ruptures to minimal trauma.

\section{Classification}

\subsection{Duke and Elders Classification [9]}

Stationary - Circular well defined opacity with central concentric ring on posterior capsule, giving a bull's eye appearance. It may or may not be associated with a satellite rosette lesion or nuclear sclerosis. The stationary type is compatible with good visual acuity.

Progressive - Characterised by radiating rider type of opacity in posterior cortex. Symptoms are more in this type of posterior polar cataract.

\subsection{Singhs Classification [10]}

Type 1: the posterior polar opacity is associated with posterior subcapsular cataract.

Type 2: sharply defined round or oval opacity with ringed appearance like an onion with or without grayish spots at the edge.

Type 3: sharply defined round or oval white opacity with dense white spots at the edge often associated with thin or absent posterior capsule. These dense white spots are a diagnostic sign (Daljit Singh sign) of posterior capsule leakage with or without repair and extreme fragility, the incidence of this type in Indian adult cataract patient population was found to be about one in 300 .

Type 4: combination of the above 3 types with nuclear sclerosis.

Singh observed the possibility of conversion of posterior polar cataract from type two to type three over following patients who for many years were reluctant to have surgery. He recommended not to delay surgery unnecessarily in these cases.

\subsection{Schroeders Classification [11]}

This classification grades posterior polar cataract in pediatric patients according to its effect in pupillary obstruction in the red reflex testing as follows:

Grade 1: a small opacity without any effect on the optical quality of the clear part of the lens.

Grade 2: a two-thirds obstruction without other effect. 
Grade 3: the disc-like opacity in the posterior capsule is surrounded by an area of further optical distortion. Only the dilated pupil shows a clear red reflex surrounding this zone.

Grade 4: the opacity is totally occlusive; no sufficient red reflex is obtained by dilation of the pupil.

He advocated for patching before surgery as it can play a diagnostic role apart from its importance for the postoperative management. If patching works well, surgery is not urgent; if not, it should be done soon. His retinoscopic grading that was mentioned above would help in timely planning of surgery. The higher the grade of pupillary obstruction the earlier the surgery should be carried out.

\subsection{Clinical Features}

Posterior polar cataract patients presents with defective vision and glare.

\subsection{Diagnosis}

On slit-lamp examination the pathognomonic feature of PPC is bulls eye appearance. Examination of the anterior vitreous may reveal oil-like droplets or particles [5] and the presence of these should raise the possibility of pre-existing posterior capsular opening. It can be associated with other ocular features like microphthalmia, microcornea, anterior polar cataract [12]. In addition, it has been found to be associated with ectodermal dysplasia, Rothmund disease, scleroderma, incontinentia pigmenti, congenital dyskeratosis, congenital ichthyosis and psychosomatic disorders (Figure 1 and Figure 2).

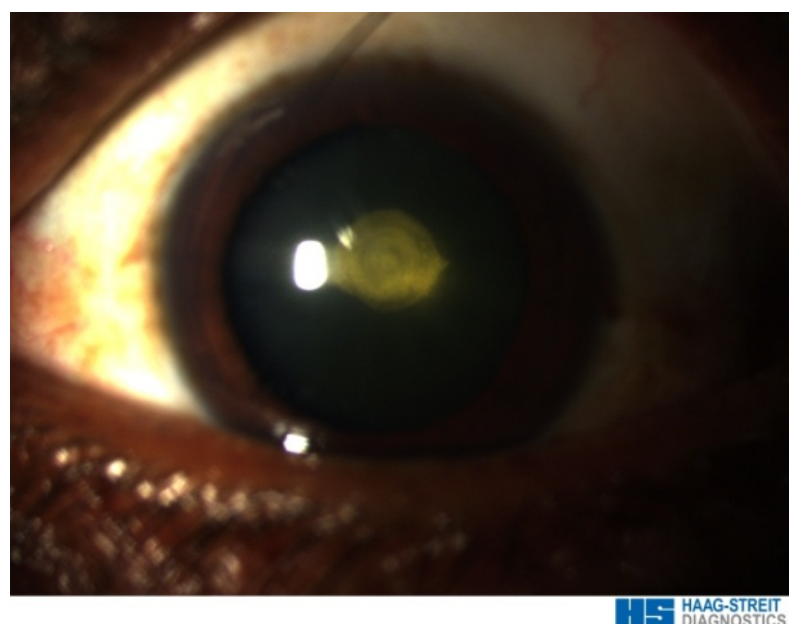

Figure 1. Bulls eye appearance of ppc in diffuse.

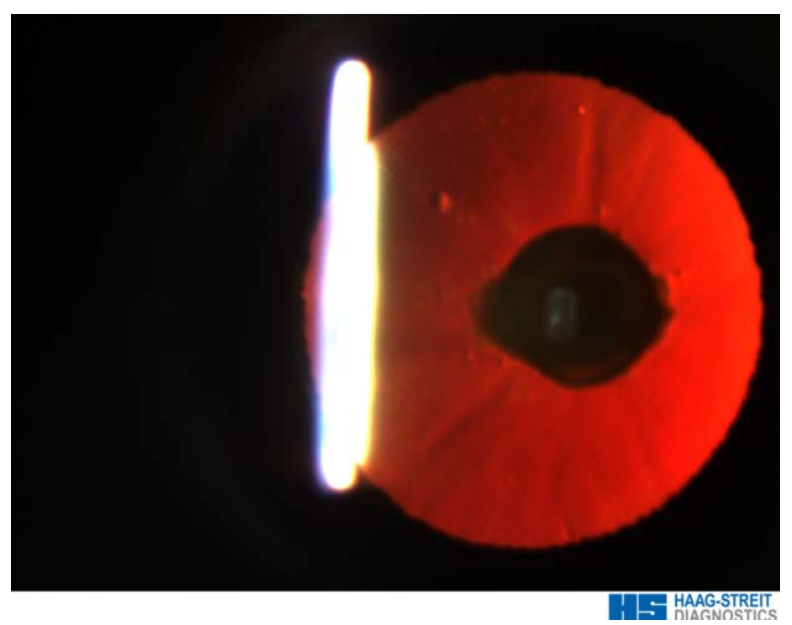

Figure 2. Appearance of ppc in retroillumination. 


\subsection{Decision to Operate}

Most of the surgeons opine that surgery should be delayed as long as possible and undertaken only when patients find difficulty in performing routine activities. However, when it is visually significant in childhood, it is considered amblyogenic which warrants an early intervention in these cases.

Counselling-During the preoperative examination, the physician should inform the patient of the possibility of the nucleus' drop intraoperatively, posterior capsular rupture, a relatively long operative time, secondary posterior segment intervention, and a delayed visual recovery. In addition, the surgeon should discuss Nd:YAG capsulotomy for residual plaque [1] [2] and emphasize the possibility of preexisting amblyopia, especially in cases of unilateral posterior polar cataract. Because the understanding of posterior polar cataract, an autosomal dominant condition, genetic counseling for the parents in addition to screening of family members is important.

Anaesthesia-Peribulbar anaesthesia with oculopressure to soften the globe decreases intraoperative positive pressure [2].

\subsection{Surgical Techniques}

While Osher et al. [1] found no difference in the rate of posterior capsule rupture between phacoemulsification and extracapsular cataract extraction (ECCE), Das et al. in a retrospective analysis concluded that phacoemulsification is preferred to ECCE in posterior polar cataract as they found higher rates of complications with ECCE [13]. However, they recommended ECCE in harder cataract and dense central plaques. They found out that posterior capsule rupture occurs most commonly during emulsification of the nucleus in phacoemulsification and during nucleus expression in ECCE cases. Vasavada and Singh [2] found that rupture occurs most commonly during epinucleus removal in phacoemulsification [1], while Osher et al. found it to happen during removal of the posterior polar opacity or during cleaning of the posterior capsule after plaque removal [2].

\section{Phacoemulsification}

\subsection{The Incision}

Starting with the side port incision followed by the injection of viscoelastic material might be better than starting with the main incision. This will avoid the possible chamber collapse that might predispose to premature rupture of the capsule. The incision of the phacoemulsification surgery can be a usual coaxial one, whether corneal or scleral, or it can be microincision for bimanual technique.

\subsection{Capsulorhexis}

Ideally, the capsulorhexis should be no larger than $5-5.5 \mathrm{~mm}$ because a larger opening may not leave adequate support for sulcus fixated intraocular lens in case the posterior capsule is compromised. Although a size of $4 \mathrm{~mm}$ or less could be detrimental if the surgeon must prolapse the nucleus into the anterior chamber [2] [14].

\subsection{Hydro Procedure}

Selection of the proper hydroprocedure is one of the most important steps in the management of posterior polar cataract. Most of the authors considered the cortical-cleaving hydrodissection [14] as an absolute contraindication in posterior polar cataract cases, as it can lead to hydraulic rupture \& can precipitate an instant nucleus drop. While Fine et al. [14] did hydrodissection in multiple quadrants with tiny amount of fluid without allowing the wave to transmit across the posterior capsule.

\subsection{Hydrodelineation}

Hydrodelineation is the most preferred hydro procedure. It helps to separate the endonucleus from the epinucleus \& prevents directly disturbing the posterior capsule. It also creates a mechanical cushion of epinucleus which acts as a safeguard. It is worth mentioning that the surgeon should avoid vigorous decompression of the capsular bag after the delineation.

\subsection{Inside-Out Hydrodelineation}

Vasavada and Raj described a technique that was described for dense and posterior polar cataract called in- 
side-out delineation [15]. In this technique, a trench is first sculpted and a right-angled cannula is used to subsequently direct fluid perpendicularly to the lens fibers in the desired plane through one wall of the trench. This would avoid the possibility of inadvertent subcapsular injection and overcome the difficulty of introducing cannula to a significant depth in a dense cataract.

\subsection{Rotation}

It should be avoided, because attempt to rotate the nucleus can lead to posterior capsule rupture [2].

\subsection{Phacoemulsification Parameters}

According to studies done, slow motion phacoemulsification with low parameters should be used. The low vacuum and aspiration rates maintain a very stable chamber and the reduced infusion drives less fluid around the lens.

\subsection{Nucleotomy Technique}

Bimanual cracking and division of the nucleus involve outward movements and can distort the capsular bag. For nuclear sclerosis $>-2$ step by step chop in situ and lateral separation technique for chopping should be used. The resultant fragments are removed with a stop and chop and stuff technique (4). For soft nucleus, the entire nucleus can be aspirated within the epinuclear shell. Lee and Lee [3] sculpted the nucleus in the shape of the Greek letter lambda " $\lambda$ technique", then cracking along both arms and removing the distal central piece. The advantage of this is its gentleness in not stretching the capsule while removing the quadrants, especially the first one.

\subsection{Epinucleus Removal}

Removing the epinucleus is again the critical step, the central plaque should be uprooted last to prevent any epinuclear fragments from falling into the vitreous cavity. First the peripheral lower half of epinucleus should be striped off, central area of epinucleus remains attached. Next, the peripheral upper epinucleus (subincisional) can be mobilized with gentle, focal, multiquadrant hydrodissection using a right-angled cannula that faces right and left [15]. Some surgeons have suggested performing viscodissection of the epinucleus by injecting viscoelastic under the capsular edge to mobilize rim of epinucleus. If posterior plaque firmly adherent to capsule is uncovered it is left behind which can be dealt with ND:YAG laser capsulotomy post operatively. Although few surgeons prefer primary posterior capsulorhexis [15] before lens implantation.

\subsection{Posterior Capsular Dehiscence}

Recognising the early signs of posterior capsule rupture during cataract surgery and its early management can lead to good visual outcomes.

- Signs of an early posterior capsular dehiscence or zonular dehiscence includes sudden deepening of the anterior chamber with momentarily expansion of pupil;

- Sudden transient appearance of clear red reflex peripherally;

- Newly apparent inability to rotate previously mobile nucleus;

- Excessive lateral displacement of nucleus and partial descent of nucleus into anterior vitreous face.

Nucleus drop-Retained posterior nuclear fragments greatly increase the risk of postoperative complications and usually must be retrieved subsequently by posterior segment approach. The likelihood of a dropped nucleus increases the longer the posterior capsule rupture goes unrecognized. The detection allow the surgeon the opportunity to convert to a large incision or extra capsule cataract extraction. A brunescent nucleus may abruptly and rapidly sink through the liquefied vitreous without antecedent vitreous loss. However, if enough supporting formed vitreous is present the nucleus will descend only partially, allowing time for rescue manoeuveres. The worst strategy for recovering a descending nucleus is to try to chase and spear the nucleus propelling the nucleus away. Attempting to phacoemulsify or aspirate it may snag vitreous in the tip, which could lead to giant retinal tears or a retinal detachment. 


\subsection{IOL Implantation}

Before implantating the IOL, viscoelastic substance is injected in to the capsular bag to expand it adequately. But it is important not to over pressurize the eye. The IOL is carefully implanted, taking care to avoid contact with the posterior capsule during the implantation. Residual viscoelastic material is removed at the end. In case of PC rent, before placing the posterior chamber IOL in the ciliary sulcus, estimate the sulcus diameter by measuring the horizontal white-to-white corneal diameter. if necessary, an attempt can be made to convert the posterior defect into a posterior capsulorhexis [3], perform a central vitrectomy then implant the IOL in the ciliary sulcus and perform the optic capture in the bag (The Rhexis-Fixated Lens) [15]. In case of PC rent, after implanting pciol the viscoelastic should be removed.

Bimanual Phacoemulsification [14] [16] — Bimanual phaco is very good option for posterior polar cataracts to minimize the risk of complications allowing posterior polar cataract extraction to be performed more safely. The added advantages of bimanual phacoemulsification are

- A controlled operating environment for slow motion phaco by virtue of its low fluidics;

- Allowing withdrawal of phaco needle first while maintaining the anterior chamber with infusion from the separate irrigating chopper.

\subsection{Modified Epinucleus Prechop for Dense Polar Cataract}

For management of dense posterior polar cataracts, anterior epinucleus is first pre chopped in piecemeal in situ maneuver before mobilizing, segmenting, and emulsifying the dense endonucleus [17]. This is followed by the removal of the posterior epinucleus and posterior polar plaque. Because chopper is repositioned at different meridians in the mid-periphery of the anterior epinucleus, it stops short of the central posterior epinucleus, thus avoiding extension of the crack toward the posterior polar plaque and posterior capsule.

Prognosis-Prognosis of cataract surgery is good if it is done carefully.

\section{Conclusion}

Though posterior polar cataract is a challenge for cataract surgeons, patience coupled with meticulous attention to the technique, which includes carefully sized capsulorhexis, avoidance of cortical cleaving hydrodissection, gentle hydrodelineation, atraumatic nucleus handling and tackling the central epinuclear plate in the last part of cortical clean up, ensures a safe surgery and favourable postoperative outcomes.

\section{References}

[1] Osher, R.H., Yu, B.C. and Koch, D.D. (1990) Posterior Polar Cataracts: A Predisposition to Intraoperative Posterior Capsular Rupture. Journal of Cataract Refractive Surgery, 16, 157-162. http://dx.doi.org/10.1016/S0886-3350(13)80724-9

[2] Vasavada, A.R. and Singh, R. (1999) Phacoemulsification with Posterior Polar Cataract. Journal of Cataract Refractive Surgery, 25, 238-245. http://dx.doi.org/10.1016/S0886-3350(99)80133-3

[3] Lee, M.W. and Lee, Y.C. (2003) Phacoemulsification of Posterior Polar Cataracts-A Surgical Challenge. British Journal of Ophthalmology, 87, 1426-1427. http://dx.doi.org/10.1136/bjo.87.11.1426-a

[4] Vogt, G., Horvath-Puho, E. and Czeizel, E. (2006) A Population-Based Case-Control Study of Isolated Congenital Cataract. Orv Hetil, 147, 1077-1084.

[5] (1924) Congenital Anomalies of the Lens as Seen with the Slit Lamp. American Journal of Ophthalmology, 7, 678-680. http://dx.doi.org/10.1016/S0002-9394(24)90741-0

[6] Addison, P.K., Berry, V., Ionides, A.C., Francis, P.J., Bhattacharya, S.S. and Moore, A.T. (2005) Posterior Polar Cataract Is the Predominant Consequence of a Recurrent Mutation in the PITX3 Gene. British Journal of Ophthalmology, 89, 138-141. http://dx.doi.org/10.1136/bjo.2004.053413

[7] Berry, V., Francis, P., et al. (2001) Alpha-B Crystallin Gene (CRYAB) Mutation Causes Dominant Congenital Posterior Polar Cataract in Humans. The American Journal of Human Genetics, 69, 1141-1145. http://dx.doi.org/10.1086/324158

[8] Eshaghian, J. and Streeten, B.W. (1980) Human Posterior Subcapsular Cataract: An Ultrastructural Study of the Posterioly Migrating Cells. Archives of Ophthalmology, 98, 134-143. http://dx.doi.org/10.1001/archopht.1980.01020030136016 
[9] Duke-Elder, S. (1964) Posterior Polar Cataract, System of Ophthalmology, Vol. iii, pt. 2: Normal and Abnormal Development, Congenital Deformities. MO, CV Mosby, St. Louis, 723-726.

[10] Masket, S. (1997) Consultation Section: Cataract Surgical Problem. Journal of Cataract and Refractive Surgery, 23, 819-824. http://dx.doi.org/10.1016/S0886-3350(97)80236-2

[11] Schroeder, H.W. (2005) The Management of Posterior Polar Cataract: The Role of Patching and Grading. Strabismus, 13, 153-156. http://dx.doi.org/10.1080/09273970500387753

[12] Harman, N.B. (1909) New Pidegrees of Cataract-Posterior Polar, Anterior Polar and Microphthalmia, and Lamellar. Transactions of the Ophthalmological Societies of the United Kingdom, 29, 296-306.

[13] Das, S., Khanna, R., Mohiuddin, S.M. and Ramamurthy, B. (2008) Surgical and Visual Outcomes for Posterior Polar Cataract. British Journal of Ophthalmology, 92, 1476-1478. http://dx.doi.org/10.1136/bjo.2007.129403

[14] Fine, I.H., Packer, M. and Hoffman, R.S. (2003) Management of Posterior Polar Cataract. Journal of Cataract \& Refractive Surgery, 29, 16-19. http://dx.doi.org/10.1016/S0886-3350(02)01616-4

[15] Vasavada, A.R. and Raj, S.M. (2004) Inside-Out Delineation. Journal of Cataract \& Refractive Surgery, 30, 11671169. http://dx.doi.org/10.1016/j.jcrs.2003.10.034

[16] Haripriya, A., Aravind, S., Vadi, K. and Natchiar, G. (2006) Bimanual Microphaco for Posterior Polar Cataracts. Journal of Cataract \& Refractive Surgery, 32, 914-917. http://dx.doi.org/10.1016/j.jcrs.2006.02.049

[17] Lim, Z. and Goh, J. (2008) Modified Epinucleus Pre-Chop for the Dense Posterior Polar Cataract. Ophthalmic Surgery, Lasers and Imaging Retina, 39, 171-173. http://dx.doi.org/10.3928/15428877-20080301-11 